\title{
A NEW PROOF OF THE WIENER-HOPF FACTORIZATION VIA BASU'S THEOREM
}

\author{
BRIAN FRALIX ${ }^{* * *}$ AND \\ COLIN GALLAGHER, ${ }^{*}$ Clemson University
}

\begin{abstract}
We illustrate how Basu's theorem can be used to derive the spatial version of the WienerHopf factorization for a specific class of piecewise-deterministic Markov processes. The classical factorization results for both random walks and Lévy processes follow immediately from our result. The approach is particularly elegant when used to establish the factorization for spectrally one-sided Lévy processes.
\end{abstract}

Keywords: Wiener-Hopf factorization; Basu's theorem; Wiener's Tauberian theorem; Lévy process

2010 Mathematics Subject Classification: Primary 60G50; 60G51

\section{Introduction}

Lévy processes are widely used for modeling purposes in many areas of applied probability, such as queueing theory, insurance risk theory, and mathematical finance. One result that has proven to be useful in such settings is known as the Wiener-Hopf factorization; here we state the spatial version only. Suppose that $X:=\{X(t) ; t \geq 0\}$ represents a Lévy process, with $X(0)=0$, and let $e_{q}$ be an exponential random variable with rate $q>0$, where $e_{q}$ is independent of $X$.

Theorem 1. (Wiener-Hopf factorization.) For a Lévy process $X:=\{X(t) ; t \geq 0\}$, the random variables $\inf _{0 \leq s \leq e_{q}} X(s)$ and $X\left(e_{q}\right)-\inf _{0 \leq s \leq e_{q}} X(s)$ are independent. Hence, for each $\omega \in \mathbb{R}$,

$$
\mathrm{E}\left[\mathrm{e}^{\mathrm{i} \omega X\left(e_{q}\right)}\right]=\mathrm{E}\left[\mathrm{e}^{\mathrm{i} \omega \inf _{0 \leq s \leq e q} X(s)}\right] \mathrm{E}\left[\mathrm{e}^{\mathrm{i} \omega\left(X\left(e_{q}\right)-\inf _{0 \leq s \leq e q} X(s)\right)}\right] .
$$

We see that the distribution of $X$ at time $e_{q}$ can be expressed as an independent sum of two random variables. Note that one of these random variables is equal in distribution to the reflected Lévy process at time $e_{q}$, which has a tractable characteristic function if inf $\operatorname{lus}_{0 \leq e_{q}} X(s)$ has one as well.

Two relatively simple proofs of the factorization have recently appeared in the literature: see [13] and [15]. Both of these papers make use of arguments from martingale theory. In [13] the Kella-Whitt martingale [10] is used to derive the factorization for a spectrally one-sided Lévy process, and the authors of [15] showed how a more elaborate martingale approach can be used to establish the factorization for an arbitrary Lévy process.

We take a different approach. Here we illustrate how Basu's theorem can be used to establish the factorization for a particular subclass of piecewise-deterministic Markov processes (PDMPs). This argument does not make use of any concepts from stochastic calculus: only

Received 5 October 2010; revision received 14 February 2012.

* Postal address: Department of Mathematical Sciences, Clemson University, O-110 Martin Hall, Box 340975, Clemson, SC 29634, USA.

** Email address: bfralix@clemson.edu 
Basu's theorem and Wiener's Tauberian theorem are needed. A well-known approximation approach can then be used to derive the factorization for both random walks and Lévy processes. Readers will also find that in some cases such an approximation argument is not needed: we will show how Basu's theorem gives a very quick proof of the Wiener-Hopf factorization for spectrally one-sided Lévy processes, without the need of an approximation step.

This paper is organized as follows. Section 2 contains a brief review of the necessary concepts needed from mathematical statistics. In Section 3 we present the proof of the main result, and we follow in Section 4 by considering the special setting of spectrally one-sided Lévy processes.

\section{Preliminaries}

We will make use of the following standard concepts from mathematical statistics: a simple introduction can be found in [2].

Suppose that we have a random sample $\left\{Q_{i}\right\}_{i=1}^{n}$, and let $U$ and $V$ be statistics with respect to the sample, i.e. both $U$ and $V$ are measurable functions of the sample. We are interested in estimating an unknown parameter $\theta \in \Theta$ (the parameter space), which appears in the distribution of the random sample. We use the measure $\mathrm{P}_{\theta}$ to emphasize that, under $\mathrm{P}_{\theta}$, the unknown parameter of the data is $\theta$.

Definition 1. We say that $U$ is a sufficient statistic with respect to $\theta$ if the conditional joint distribution of the data $\left\{Q_{i}\right\}_{i=1}^{n}$, given $U$, is independent of $\theta$.

Definition 2. We say that $V$ is an ancillary statistic if its distribution does not depend on $\theta$.

Definition 3. We say that $U$ is a boundedly complete statistic if it satisfies the following criterion: if $g$ is a bounded measurable function which satisfies $\mathrm{E}_{\theta}[g(U)]=0$ for all $\theta \in \Theta$ then $\mathrm{P}_{\theta}(g(U)=0)=1$ for each $\theta \in \Theta$.

We are now ready to state Basu's theorem.

Theorem 2. (Basu's theorem.) Suppose that $U$ is a boundedly complete, sufficient statistic with respect to $\theta \in \Theta$, and suppose that $V$ is an ancillary statistic with respect to $\theta \in \Theta$. Then $U$ and $V$ are independent under $\mathrm{P}_{\theta}$ for each $\theta \in \Theta$.

The proof of Basu's theorem is simple, and can be found in many textbooks on mathematical statistics: see, for example, [2]. Basu's theorem is also featured in the recent survey articles of Boos and Hughes-Oliver [1] and Ghosh [6], where classical and new applications are discussed in both papers.

The applications of Basu's theorem in our study will always involve a given stochastic process $\{X(t) ; t \geq 0\}$ and an independent exponential random variable $e_{q}$ with rate $q>0$. Our data will always be $\left(\inf _{0 \leq s \leq e_{q}} X(s), X\left(e_{q}\right)-\inf _{0 \leq s \leq e_{q}} X(s)\right)$, with the two statistics of interest being the two coordinates of the data. The unknown parameter is always the initial condition $X(0)=\theta$, and the measure $\mathrm{P}_{\theta}$ will always represent conditioning on $X(0)=\theta$, as is typically done in the theory of Markov processes.

\section{Our main result}

Our process of interest in this section is a PDMP $X:=\{X(t) ; t \geq 0\}$, which evolves as follows. The process $X$ has jumps at the random locations $\left\{T_{k}\right\}_{k \geq 1}$, which also form the points of an ordinary renewal process. The size of the jump at location $T_{k}$ is $B_{k}, k \geq 1$, and we assume 
that the $\left\{B_{k}\right\}_{k \geq 1}$ sequence is independent and identically distributed (i.i.d.), and independent of the jump locations. Between jumps, $X$ moves in a deterministic manner according to a nonnegative function $h$, defined on the real line.

A more specific description of the evolution of $X$, starting at a deterministic $X(0)=\theta$, is as follows: for $0 \leq t<T_{1}, X(t)=X(0)+h(t)$ and $X\left(T_{1}\right)=X\left(T_{1}-\right)+B_{1}$ with $X(t-)=\lim _{s \uparrow t} X(s)$ for each $t \geq 0$. Similarly, for $T_{1} \leq t<T_{2}, X(t)=X\left(T_{1}\right)+h\left(t-T_{1}\right)$, $X\left(T_{2}\right)=X\left(T_{2}-\right)+B_{2}$, and, for an arbitrary $n \geq 1, X(t)=X\left(T_{n}\right)+h\left(t-T_{n}\right)$ for $T_{n} \leq t<$ $T_{n+1}$, with $X\left(T_{n+1}\right)=X\left(T_{(n+1)}-\right)+B_{n+1}$.

We further assume that there exists a sequence of left-continuous functions $\left\{h_{n}\right\}_{n \geq 0}$ such that (i) $h_{n}:[0, \infty) \rightarrow\left\{k / 2^{n}: k \geq 0\right\}$, and (ii) the sequence $\left\{h_{n}\right\}_{n \geq 1}$ converges uniformly to $h$ on compact sets as $n \rightarrow \infty$. These functions are used to define a collection of approximating PDMPs $\left\{X^{n}\right\}_{n \geq 0}$. For each $n \geq 0, X^{n}$ is a PDMP that is governed by the renewal process $N$, the function $h_{n}$, and the set of jump sizes $\left\{B_{n, k}\right\}_{k \geq 1}$, where, for each $k \geq 1$,

$$
B_{n, k}=\frac{\inf \left\{l \in \mathbb{Z}: l / 2^{n} \geq B_{k}\right\}}{2^{n}} .
$$

We also assume that the initial value $X^{n}(0)=\theta_{n} \in\left\{k / 2^{n} ; k \in \mathbb{Z}\right\}$ is such that $\theta_{n} \rightarrow \theta$ as $n \rightarrow \infty$. Note that at each time instant $t \geq 0, X^{n}(t)$ takes values in the space $\left\{k / 2^{n} ; k \in \mathbb{Z}\right\}$.

We now establish that $\inf _{0 \leq s \leq e_{q}} X^{0}(s)$ is both sufficient and boundedly complete.

Lemma 1. $\inf _{0 \leq s \leq e_{q}} X^{0}(s)$ is a sufficient statistic with respect to its unknown initial condition $\theta_{0} \in \mathbb{Z}$.

Proof. Let $A$ and $B$ be two subsets of the integers, and let $x$ be an arbitrary integer. Setting $\mathrm{P}_{\theta_{0}}$ as a probability measure, under which the law of $X^{0}$ is as the integer-valued PDMP described above with initial value $X^{0}(0)=\theta_{0}$, we see that, for $x \leq \theta_{0}$,

$$
\begin{gathered}
\mathrm{P}_{\theta_{0}}\left(X^{0}\left(e_{q}\right)-\inf _{0 \leq s \leq e_{q}} X^{0}(s) \in A, \inf _{0 \leq s \leq e_{q}} X^{0}(s) \in B \mid \inf _{0 \leq s \leq e_{q}} X^{0}(s)=x\right) \\
=\mathbf{1}(x \in B) \frac{\mathrm{P}_{\theta_{0}}\left(X^{0}\left(e_{q}\right) \in A+x, \inf _{0 \leq s \leq e_{q}} X^{0}(s)=x\right)}{\mathrm{P}_{\theta_{0}}\left(\inf _{0 \leq s \leq e_{q}} X^{0}(s)=x\right)} .
\end{gathered}
$$

Set $\tau_{x}=\inf \left\{t \geq 0: X^{0}(t) \leq x\right\}$. A conditioning argument gives

$$
\begin{aligned}
\mathrm{P}_{\theta_{0}}\left(X^{0}\left(e_{q}\right) \in A+x, \inf _{0 \leq s \leq e_{q}} X^{0}(s)=x\right) \\
\quad=\mathrm{P}_{\theta_{0}}\left(X^{0}\left(e_{q}\right) \in A+x, \inf _{0 \leq s \leq e_{q}} X^{0}(s)=x \mid X^{0}\left(e_{q} \wedge \tau_{x}\right)=x\right) \mathrm{P}_{\theta_{0}}\left(X^{0}\left(e_{q} \wedge \tau_{x}\right)=x\right) \\
\quad=\mathrm{P}_{x}\left(X^{0}\left(e_{q}\right) \in A+x, \inf _{0 \leq s \leq e_{q}} X^{0}(s)=x\right) \mathrm{P}_{\theta_{0}}\left(X^{0}\left(\tau_{x} \wedge e_{q}\right)=x\right)
\end{aligned}
$$

Note that we are making use of the fact that the process regenerates at the points of our renewal process $N$; moreover, since $h^{0} \geq 0$, the infimum of $X^{0}$ over any interval must be attained at a renewal instant within that interval.

A similar argument shows that

$$
\mathrm{P}_{\theta_{0}}\left(\inf _{0 \leq s \leq e_{q}} X^{0}(s)=x\right)=\mathrm{P}_{x}\left(\inf _{0 \leq s \leq e_{q}} X^{0}(s)=x\right) \mathrm{P}_{\theta_{0}}\left(X^{0}\left(\tau_{x} \wedge e_{q}\right)=x\right) .
$$


Substituting both (2) and (3) into (1) yields

$$
\begin{aligned}
\mathrm{P}_{\theta_{0}}\left(X^{0}\left(e_{q}\right)-\inf _{0 \leq s \leq e_{q}} X^{0}(s) \in A, \inf _{0 \leq s \leq e_{q}} X^{0}(s) \in B \mid \inf _{0 \leq s \leq e_{q}} X^{0}(s)=x\right) \\
=\mathbf{1}(x \in B) \frac{\mathrm{P}_{x}\left(X^{0}\left(e_{q}\right) \in A+x, \inf _{0 \leq s \leq e_{q}} X^{0}(s)=x\right) \mathrm{P}_{\theta_{0}}\left(X^{0}\left(\tau_{x} \wedge e_{q}\right)=x\right)}{\mathrm{P}_{x}\left(\inf _{0 \leq s \leq e_{q}} X^{0}(s)=x\right) \mathrm{P}_{\theta_{0}}\left(X^{0}\left(\tau_{x} \wedge e_{q}\right)=x\right)} \\
=\mathbf{1}(x \in B) \frac{\mathrm{P}_{x}\left(X^{0}\left(e_{q}\right) \in A+x, \inf _{0 \leq s \leq e_{q}} X^{0}(s)=x\right)}{\mathrm{P}_{x}\left(\inf _{0 \leq s \leq e_{q}} X^{0}(s)=x\right)},
\end{aligned}
$$

and this conditional probability does not depend on $\theta_{0}$. Hence, $\inf _{0 \leq s \leq e_{q}} X^{0}(s)$ is a sufficient statistic.

Remark 1. Note how a discrete state space setting makes proving sufficiency of $\inf _{0 \leq s \leq e_{q}} X(s)$ a simple matter. An analogous proof in a general state space setting appears to be difficult to produce, although it seems obvious that it must hold.

Our next task is to realize that $\inf _{0 \leq s \leq e_{q}} X_{0}^{0}(s)$ is infinitely divisible, where $X_{0}^{0}$ represents the PDMP $X^{0}$, with $\theta_{0}=0$. Clearly, $X^{0}(t)=X_{0}^{0}(t)+\theta_{0}$ under $\mathrm{P}_{\theta_{0}}$ for each $t \geq 0$.

Lemma 2. $\inf _{0 \leq s \leq e_{q}} X_{0}^{0}(s)$ is an infinitely divisible random variable.

Proof. The proof of this statement can be found in [5].

We now check to see if $\inf _{0 \leq s \leq e_{q}} X^{0}(s)$ is boundedly complete. To show this, we make use of Wiener's Tauberian theorem. Such an argument has been used before to verify that location families are boundedly complete; see, for example, Theorem 2.4 of [7]. In fact, the version of Wiener's Tauberian theorem used here is also applied in Example 2.6(d) of [14].

Lemma 3. $\inf _{0 \leq s \leq e_{q}} X^{0}(s)$ is boundedly complete.

Proof. The proof follows from a discrete version of Wiener's Tauberian theorem; see [8, p. 71]. This theorem tells us that, since the characteristic function of $\inf _{0 \leq s \leq e_{q}} X_{0}^{0}(s)$ does not vanish (because of infinite divisibility), we may conclude that any absolutely summable sequence $x=\{x(k)\}_{k \in \mathbb{Z}} \in \ell_{1}$ can be approximated under the $\ell_{1}$-norm by elements $\mathcal{A} \in \ell_{1}$ of the form

$$
\mathcal{A}(j)=\sum_{k=1}^{m} a_{k} p_{0}\left(j+c_{k}\right),
$$

where $p_{0}$ represents the probability mass function of $\inf _{0 \leq s \leq e_{q}} X_{0}^{0}(s), m$ is an arbitrary positive integer, $\left\{a_{k}\right\}_{k=1}^{m}$ is a sequence of complex numbers, and $\left\{c_{k}\right\}_{k=1}^{m}$ is a collection of integers.

Fix a bounded, measurable function $g: \mathbb{Z} \rightarrow \mathbb{R}$ satisfying $\mathrm{E}_{\theta}\left[g\left(\inf _{0 \leq s \leq e_{q}} X^{0}(s)\right)\right]=0$ for all $\theta \in \mathbb{Z}$, and let $F: \ell_{1} \rightarrow \mathbb{R}$ be a function satisfying, for each $x \in \ell_{1}$,

$$
F(x)=\sum_{k \in \mathbb{Z}} g(k) x(k) .
$$

Continuity of $F$ under the $\ell_{1}$-norm yields $F(x)=0$ for each $x \in \ell_{1}$, since we can find a sequence $\left\{x_{n}\right\}_{n \geq 1}$ of linear combinations of the form (4), satisfying $F\left(x_{n}\right)=0$ for all $n$, that converge to $x$. Thus, $g(n)=0$ for each integer $n$, which completes the proof.

We conclude the proof of the factorization by showing that $X^{0}\left(e_{q}\right)-\inf _{0 \leq s \leq e_{q}} X^{0}(s)$ is an ancillary statistic. 
Lemma 4. $X^{0}\left(e_{q}\right)-\inf _{0 \leq s \leq e_{q}} X^{0}(s)$ is an ancillary statistic.

Proof. The proof of this result is straightforward: note that

$$
X^{0}\left(e_{q}\right)-\inf _{0 \leq s \leq e_{q}} X^{0}(s)=\theta_{0}+X_{0}^{0}\left(e_{q}\right)-\inf _{0 \leq s \leq e_{q}}\left(\theta_{0}+X_{0}^{0}(s)\right)=X_{0}^{0}\left(e_{q}\right)-\inf _{0 \leq s \leq e_{q}} X_{0}^{0}(s),
$$

which does not depend on $\theta_{0}$.

Theorem 3. $\inf _{0 \leq s \leq e_{q}} X(s)$ is independent of $X\left(e_{q}\right)-\inf _{0 \leq s \leq e_{q}} X(s)$, where $e_{q}$ is an exponential random variable with rate $q>0$, independent of $X^{0}$.

Proof. Combining Basu's theorem with our previous lemmas shows that $\inf _{0 \leq s \leq e_{q}} X^{0}(s)$ is independent of $X^{0}\left(e_{q}\right)-\inf _{0 \leq s \leq e_{q}} X^{0}(s)$. From our proof technique, it is clear that, for each $n \geq 1,2^{n} \inf _{0 \leq s \leq e_{q}} X^{n}(s)$ is both sufficient and boundedly complete, and so $\inf _{0 \leq s \leq e_{q}} X^{n}(s)$ is independent of $X^{n}\left(e_{q}\right)-\inf _{0 \leq s \leq e_{q}} X^{n}(s)$. Finally, since $f_{n}$ converges uniformly on compact sets to $f$ as $n$ gets large, $\inf _{0 \leq s \leq e_{q}} X(s)$ is independent of $X\left(e_{q}\right)-\inf _{0 \leq s \leq e_{q}} X(s)$, because, with probability 1 , the sample paths of $X^{n}$ converge uniformly on compact sets to the sample paths of $X$.

Remark 2. It was shown in [5] that $\inf _{0 \leq s \leq e_{q}} X_{0}^{0}\left(e_{q}\right)$ is infinitely divisible, by making use of the fact that this random variable can be expressed as a geometric sum of i.i.d. undershoot variables. Some readers may find this argument unsatisfactory, as the main idea used to verify that this is an i.i.d. geometric sum seems to be quite close to the decomposition approach used in the random walk proof of the Wiener-Hopf factorization found in, for example, the proof of Theorem 1 of [12], which was inspired by the work of Greenwood and Pitman [9]. This decomposition approach, in the random walk setting, can also be used to establish the required independence property.

Nevertheless, we still feel that our approach should be of interest to the applied probability community, as we can use any proof we like to show that the characteristic function of $\inf _{0 \leq s \leq e_{q}} X_{0}^{0}(s)$ does not vanish, and, for some models, this may be much easier to do by other methods; one example will be given in the next section.

We conclude this section with a brief explanation of how this result carries over to the Lévy setting.

Theorem 4. Suppose that $X:=\{X(t) ; t \geq 0\}$ represents a Lévy process with characteristic triplet $\left(a, \sigma^{2}, \pi\right)$, which we assume to be known. Then $\inf _{0 \leq s \leq e_{q}} X(s)$ is independent of $X\left(e_{q}\right)$ $\inf _{0 \leq s \leq e_{q}} X(s)$.

Proof. Any continuous-time Markov chain that is also a Lévy process satisfies the WienerHopf factorization, as these processes are within our subclass of PDMPs. A two-step scaling procedure can be used to show how this result extends to all types of Lévy process; details can be found in Section 4 of [5].

\section{Spectrally one-sided Lévy processes}

In some instances, it is possible to use Basu's theorem to establish the factorization without having to use an approximation argument, undershoot variables, or a Tauberian theorem.

Suppose that $X:=\{X(t) ; t \geq 0\}$ is a spectrally positive Lévy process, with characteristic triplet $\left(a, \sigma^{2}, \pi\right)$ and unknown (deterministic) initial condition $X(0)=\theta$ : saying that $X$ is 
spectrally positive means that the support of the Lévy jump measure $\pi$ is contained in $[0, \infty)$, i.e. the process has no negative jumps. It is well known that in this setting, under $\mathrm{P}_{\theta}$,

$$
\inf _{0 \leq s \leq e_{q}} X(s) \stackrel{\mathrm{D}}{=} \theta-E,
$$

where $E$ is an exponential random variable with rate $\Phi(q)$, with $\Phi$ being a suitable inverse function; see Section 3.3 of [11] for details. This leads to the following proposition.

Proposition 1. $\inf _{0 \leq s \leq e_{q}} X(s)$ is a boundedly complete, sufficient statistic with respect to $\theta$.

Proof. Proving that $\inf _{0 \leq s \leq e_{q}} X(s)$ is a sufficient statistic with respect to $\theta$ is relatively simple, since the process has no negative jumps: here, we can make use of the strong Markov property and the memoryless property of $e_{q}$ to conclude that, under $\mathrm{P}_{\theta}$, for $x<\theta$,

$$
\begin{aligned}
\mathrm{P}_{\theta}\left(X\left(e_{q}\right)-\inf _{0 \leq s \leq e_{q}} \in A, \inf _{0 \leq s \leq e_{q}} X(s) \in B \mid \inf _{0 \leq s \leq e_{q}} X(s)=x\right) \\
=\mathbf{1}(x \in B) \lim _{h \downarrow 0} \frac{\mathrm{P}_{\theta}\left(X\left(e_{q}\right)-\inf _{0 \leq s \leq e_{q}} X(s) \in A, \inf _{0 \leq s \leq e_{q}} X(s) \in[x, x+h]\right)}{\mathrm{P}_{\theta}\left(\inf _{0 \leq s \leq e_{q}} X(s) \in[x, x+h]\right)} \\
=\mathbf{1}(x \in B) \lim _{h \downarrow 0} \frac{\mathrm{P}_{x+h}\left(X\left(e_{q}\right)-\inf _{0 \leq s \leq e_{q}} X(s) \in A, \inf _{0 \leq s \leq e_{q}} X(s) \in[x, x+h]\right)}{\mathrm{P}_{x+h}\left(\inf _{0 \leq s \leq e_{q}} X(s) \in[x, x+h]\right)},
\end{aligned}
$$

and this conditional probability does not depend on $\theta$. Hence, $\inf _{0 \leq s \leq e_{q}} X(s)$ is a sufficient statistic of $\left(X\left(e_{q}\right)-\inf _{0 \leq s \leq e_{q}} X(s), \inf _{0 \leq s \leq e_{q}} X(s)\right)$ with respect to $\theta$. Readers wishing to see a reference on regular conditional distributions that explains the limiting step given above are referred to [4].

We now show that $\inf _{0 \leq s \leq e_{q}} X(s)$ is boundedly complete. Suppose that $g$ is a bounded Borel measurable function, satisfying $\mathrm{E}_{\theta}\left[g\left(\inf _{0 \leq s \leq e_{q}} X(s)\right)\right]=0$ for all $\theta \in \mathbb{R}$. Then, for each $\theta$,

$$
0=\mathrm{E}_{\theta}\left[g\left(\inf _{0 \leq s \leq e_{q}} X(s)\right)\right]=\int_{-\infty}^{\theta} g(u) \Phi(q) \mathrm{e}^{-\Phi(q)(\theta-u)} \mathrm{d} u .
$$

However, $\mathrm{e}^{-\Phi(q) \theta}>0$ for each $\theta$, which gives

$$
0=\int_{-\infty}^{\theta} g(u) \Phi(q) \mathrm{e}^{\Phi(q) u} \mathrm{~d} u,
$$

and so we conclude that $g(u)=0$ for almost all $u$, which proves the claim.

Finally, it is now obvious that $X\left(e_{q}\right)-\inf _{0 \leq s \leq e_{q}} X(s)$ is an ancillary statistic, so we may conclude that the Wiener-Hopf factorization holds.

\section{Final remarks}

Recently, DasGupta [3] showed how Basu's theorem can be used to prove that a fairly large subclass of random variables is infinitely divisible. Interestingly, he commented that 'the possible horizon of applications of Basu's theorem is probably much wider than has been understood so far.' Our approach to the Wiener-Hopf factorization seems to further support this claim, and we believe that a proper understanding of this theorem could possibly lead to a greater understanding of other concepts in applied probability, both classical and new. 


\section{References}

[1] Boos, D. D. And Hughes-Oliver, J. M. (1998). Applications of Basu's theorem. Amer. Statistician 52, $218-221$.

[2] Casella, G. And Berger, R. L. (2002). Statistical Inference. Duxbury, Pacific Grove, CA.

[3] DasGupta, A. (2007). Extensions to Basu's theorem, factorizations, and infinite divisibility. J. Statist. Planning Infer. 137, 945-952.

[4] Feller, W. (1971). An Introduction to Probability Theory and Its Applications, Vol. II. John Wiley, New York.

[5] Fralix, B. H., van LeeuwaArden, J. S. H. and Boxma, O. J. (2011). A new Wiener-Hopf identity for a general class of reflected processes. Submitted.

[6] Gноsн, M. (2002). Basu's theorem with applications: a personalistic review. Sankhyā A 64, 509-531.

[7] Ghosh, J. K. and Singh, R. (1966). Unbiased estimation of location and scale parameters. Ann. Math. Statist. 37, 1671-1675.

[8] Goldberg, R. R. (1970). Fourier Transforms. Cambridge University Press.

[9] Greenwood, P. And Pitman, J. (1980). Fluctuation identities for Lévy processes and splitting at the maximum. Adv. Appl. Prob. 12, 893-902.

[10] Kella, O. And Whitt, W. (1992). Useful martingales for stochastic storage processes with Lévy input. J. Appl. Prob. 29, 396-403.

[11] Kyprianou, A. E. (2006). Introductory Lectures on Fluctuations of Lévy Processes with Applications. Springer, Berlin.

[12] Kyprianou, A. E. (2010). The Wiener-Hopf decomposition. In Encyclopedia of Quantitative Finance, John Wiley, Chichester.

[13] Kyprianou, A. E. ANd Palmowski, Z. (2005). A martingale review of some fluctuation theory for spectrally negative Lévy processes. In Séminaire de Probabilités XXXVIII (Lecture Notes Math. 1857), Springer, Berlin, pp. 16-29.

[14] Mattner, L. (1993). Some incomplete but boundedly complete location families. Ann. Statist. 21, $2158-2162$.

[15] NguYen-Ngoc, L. AND Yor, M. (2005). Some martingales associated to reflected Lévy processes. In Séminaire de Probabilités XXXVIII (Lecture Notes Math. 1857), Springer, Berlin, pp. 42-69. 